\title{
Fibras poliméricas como aditivos de secagem em diferentes classes de concretos refratários
}

\section{(Polymeric fibers as drying additives for different classes of refractory castables)}

\author{
R. Salomão, V. C. Pandolfelli \\ Departamento de Engenharia de Materiais - DEMa \\ Universidade Federal de S. Carlos - UFSCar \\ Rod. Washington Luiz, Km 235, C.P. 676, S. Carlos, SP 13565-905 \\ pers@iris.ufscar.br,vicpando@power.ufscar.br
}

\begin{abstract}
Resumo
Concretos refratários apresentam grande variedade de métodos de instalação, definidos principalmente pelas características da aplicação e do projeto de engenharia da microestrutura do material. Entre as classes mais importantes, destacam-se os autoescoantes, aplicados sem cisalhamento externo; os bombeáveis, transportados por tubulações, e os vibráveis. Diferenciando-se principalmente pelas mudanças de distribuição de tamanho de partículas, essas classes apresentam grande variedade de propriedades como permeabilidade, módulo de ruptura a quente e resistência à fluência, descrita na literatura. Por também apresentarem diferentes comportamentos de secagem, é razoável supor que tenham necessidades de aditivos de secagem distintas. Para verificar essa hipótese, foram estudadas três composições de concretos refratários formulados a partir do modelo de empacotamento de partículas de Andreasen: bombeável (q=0,21), autoescoante $(\mathrm{q}=0,26)$ e vibrável $(\mathrm{q}=0,31)$. Fibras poliméricas de diferentes geometrias foram adicionadas a essas composições e seu efeito como aditivo de secagem foi avaliado. Verificou-se que as fibras atuaram favoravelmente em todas as formulações, aumentando significativamente sua velocidade de secagem, com efeitos mais marcantes quanto maior o teor de finos no concreto.

Palavras-chave: concretos refratários, autoescoante, bombeável, vibrável, secagem, fibras poliméricas.
\end{abstract}

\begin{abstract}
Refractory castables present a broad variety of placing methods, defined mainly by the desired application and by the material microstructural characteristics. Among the most important, it can be mentioned the self-flowing, which are cast without external shearing; the pumpable, conveyed through metallic pipes, and the vibrated. These classes of castables present a great range of properties as permeability, hot modulus of rupture and creep resistance. As they also present particularities in their drying behavior, it is reasonable to consider that they would also have different needs of drying additives. In order to verify this hypothesis, three castables compositions were designed by the Andreasen particles packing model: self-flowing $(q=0.21)$, pumpable $(q=0.26)$ and vibrated $(q=03.1)$. Polymeric fibers of several geometries were added to these compositions and their effect as drying additives were evaluated. It was found out that the fiber addition brought benefits in all the formulations, increasing their drying rates.
\end{abstract}

Keywords: refractory castables, self-flowing, pumpable, vibrated, drying polymeric fibers.

\section{INTRODUÇÃO}

Concretos refratários vêm ganhando espaço progressivamente em relação aos refratários pré-moldados convencionais devido à sua grande flexibilidade de projeto, menores custos com mão-de-obra na instalação e variedade de propriedades e técnicas de instalação. Entre as classes mais importantes, destacam-se os bombeáveis, transportados por tubulações; os autoescoantes, aplicados sem cisalhamento externo; e os vibráveis. Entre as principais diferenças, a mudança de distribuição de tamanhos de partículas pode ser apontada como principal responsável pela grande variedade de propriedades como permeabilidade, módulo de ruptura a quente, resistência à fluência e comportamento de secagem, descritos na literatura [1-4].

Essa mudança, em geral é feita, alterando-se as proporções entre as diversas matérias primas que compõe a formulação. Nas três classes de concretos empregadas nesse estudo, essas proporções podem ser descritas simplificadamente como um balanço entre a quantidade de partículas finas, ou matriz, $\left(\mathrm{Dp}_{\text {Médio }}\right.$ $<100 \mu \mathrm{m})$ e grosseiras, ou agregados, $\left(\mathrm{Dp}_{\text {Médio }} \geq 100 \mu \mathrm{m}\right)$. As formulações bombeáveis possuem uma maior proporção de matriz que as autoescoantes e vibráveis (valores típicos: $45 \%$ vol contra $39 \%$ vole $31 \%$ vol, respectivamente) [2, 4]. A adequada 
distribuição granulométrica aliada à correta dispersão da matriz faz com que os concretos autoescoantes possam fluir unicamente sob atuação do próprio peso. Para os vibráveis, a presença de uma maior proporção de partículas grosseiras dificulta consideravelmente o cisalhamento do material, necessitando assim de vibração para instalar o material. Já as formulações bombeáveis, requerem uma maior proporção de finos para que apresentem características pseudoplásticas adequadas ao transporte em tubulações. A Fig. 1 mostra uma representação esquemática desse mecanismo.

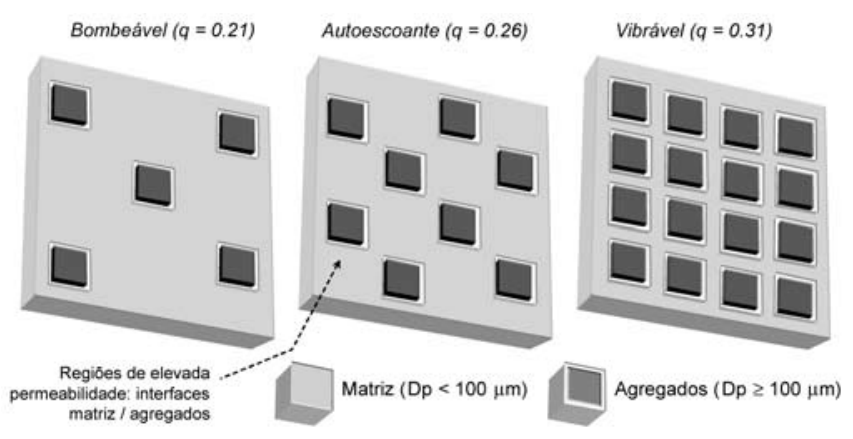

Figura 1: Representação da proporção entre partículas finas e grosseiras nos diferentes tipos de formulações de concretos refratários.

[Figure 1:Schematic view of the relationship between fine and coarse particles in castables formulations.]

Essa mudança nas proporções de finos e grossos também afeta o comportamento de secagem desses materiais $[1,4]$. Em concretos refratários, as regiões de elevada permeabilidade da estrutura estão localizadas nas regiões de interface entre matriz e agregados. Aumentando-se a quantidade dessas interfaces, aumenta-se a permeabilidade do material e sua velocidade de secagem. Devido a esse fato, é razoável supor que essas diferenças de comportamento de secagem também induzam necessidades distintas de aditivos de secagem [5-7]. Para verificar essa hipótese, foram estudadas três composições de concretos refratários formulados a partir do modelo de empacotamento de partículas de Andreasen: bombeável ( $\mathrm{q}=0,21)$, autoescoante $(\mathrm{q}=0,26)$ e vibrável $(\mathrm{q}=0,31)$. Fibras poliméricas de diferentes geometrias foram adicionadas a essas composições e seu efeito como aditivo de secagem foi avaliado.

\section{MATERIAIS E MÉTODOS:}

Foram utilizadas três composições de concreto refratário elaboradas segundo o modelo de distribuição de partículas de Andreasen, com coeficientes q iguais a 0,21 (bombeável), 0,26 (autoescoante) e 0,31 (vibrável), calculadas por meio do programa PS Designer, desenvolvido pelo Grupo de Pesquisa dos autores. As matérias-primas utilizadas compuseram uma mistura de matriz fina $\left(\mathrm{d}_{\mathrm{p}}<100 \mu \mathrm{m}\right)$, agregados $\left(\mathrm{d}_{\max }=4,75 \mathrm{~mm}\right)$ e $2 \%$ em peso de cimento de aluminato de cálcio, CA14 Almatis (EUA). Os agregados eletrofundidos foram fornecidos pela Elfusa-Brasil e as aluminas calcinadas pela Almatis-EUA. Um teor de 4,12\% em peso (15\% em volume) de água destilada foi adicionado à formulação para mistura e hidratação do cimento. Ácido cítrico foi utilizado como agente dispersante em um teor de $2,60 \times 10^{-4} \mathrm{mg} / \mathrm{m}^{2}$ em relação à área superficial dos concretos.

Tabela I: Formulações estudadas.

[Table I: Formulations tested.]

\begin{tabular}{ccccc}
\hline & & \multicolumn{3}{c}{ Formulações } \\
\cline { 3 - 5 } Matérias primas & & $\begin{array}{c}\text { Bombeável } \\
(\mathrm{q}=0,21)\end{array}$ & $\begin{array}{c}\text { Autoescoante } \\
(\mathrm{q}=0,26)\end{array}$ & $\begin{array}{c}\text { Vibrável } \\
(\mathrm{q}=0,31)\end{array}$ \\
\hline Matriz & A1000 SG & 15,00 & 10,00 & 7,00 \\
$\left(\mathrm{D}_{\max }<100 \mu \mathrm{m}\right.$ & A3000 FL & 11,20 & 8,10 & 5,00 \\
\%peso $)$ & CA14M & 2,00 & 2,00 & 2,00 \\
\hline & AEB 200F & 14,20 & 16,20 & 17,30 \\
& AEB 40F & 18,10 & 18,20 & 18,10 \\
Agregados & AEB 20/40 & 9,00 & 7,00 & 10,00 \\
$\left(\mathrm{D}_{\max } \geq 100 \mu \mathrm{m} \%\right.$ peso $)$ & AEB 10/36 & 8,00 & 11,50 & 11,50 \\
& AEB 08/20 & 9,00 & 9,00 & 11,10 \\
& AEB 04/10 & 13,50 & 18,00 & 18,00 \\
\hline Água destilada $(\%$ peso $)$ & & 4,50 & 4,50 & 4,50 \\
Área superficial $\left(\mathrm{m}^{2} / \mathrm{g}\right)$ & & 1,67 & 1,16 & 0,81 \\
Ácido cítrico $\left(\mathrm{mg} / \mathrm{m}^{2}\right)$ & & $2,60 \times 10^{-4}$ & $2,60 \times 10^{-4}$ & $2,60 \times 10^{-4}$ \\
\hline$\Phi_{\text {vol }}$ Matriz $\left(\mathrm{D}_{\max }<100 \mu \mathrm{m}, \%\right)$ & & 45,00 & 39,00 & 31,00 \\
$\Phi_{\text {Vol }}$ Agregados $\left(\mathrm{D}_{\max } \geq 100 \mu \mathrm{m}, \%\right)$ & 55,00 & 61,00 & 69,00 \\
\hline
\end{tabular}


Tabela II: Características típicas das fibras estudadas. [Table II: Characteristics of the fibers tested.]

\begin{tabular}{cc}
\hline Composição & Polipropileno \\
\hline Densidade & $0,91 \mathrm{~g} / \mathrm{cm} 3$ \\
Comprimento $(\mathrm{mm})$ & $1 \mathrm{e} 3$ \\
Diâmetro $(\mu \mathrm{m})$ & 15 \\
Teores $(\%$ peso $)[\% \mathrm{vol}]$ & $0,090[0,36]$ \\
Aplicação de origem & Aditivo de secagem em \\
& concretos refratários \\
\hline
\end{tabular}

Fibras de polipropileno de diferentes comprimentos, mesmo diâmetro e composição química foram adicionadas às formulações. Nas Tabelas I e II, as formulações de concreto e as fibras estudadas são descritas em detalhes.

A mistura foi feita em um misturador planetário sob rotação constante (33 rpm). A adição de água foi feita em uma taxa constante de $8 \mathrm{~g} / \mathrm{s}$, primeiramente, adicionando-se a água necessária para atingir o ponto de virada ( $70 \%$ do total), seguindo-se do restante, após fim do ponto de virada. Após a homogenização, as composições foram moldadas na forma de cilindros de $40 \times 40 \mathrm{~mm}$, para os ensaios de secagem, e $25 \mathrm{~mm}$ de altura por $70 \mathrm{~mm}$ de diâmetro, para as medidas de permeabilidade. Durante a moldagem, termopares tipo $\mathrm{K}$ foram inseridos, posicionados junto à superfície, na meia altura do cilindro. A cura das amostras foi feita a $8{ }^{\circ} \mathrm{C}$, numa câmara climatizada (Vötsch 2020), em ambiente saturado de umidade, por 15 dias.

A magnitude do aumento de permeabilidade gerado pelos diferentes tipos de fibras foi medida em amostras verdes e após tratamento térmico usando as constantes de permeabilidade Darciana $\left(\mathrm{k}_{1}\right)$ e não-Darcyana $\left(\mathrm{k}_{2}\right)$ [8]. Elas foram calculadas usando ajuste polinominal de dados de pressão e taxa de fluxo à Equação de Forchheimer (Equação A), expressa para fluidos compressíveis como:

$$
\frac{\mathrm{P}_{\mathrm{i}}^{2}-\mathrm{P}_{0}^{2}}{2 \mathrm{P}_{0} \mathrm{~L}}=\frac{\mu}{\mathrm{k}_{1}} \mathrm{v}_{\mathrm{s}}+\frac{\rho}{\mathrm{k}_{2}} \mathrm{v}_{\mathrm{s}}^{2}
$$

$\mathrm{P}_{\mathrm{i}}$ e $\mathrm{P}_{\mathrm{O}}$ são, respectivamente, a pressão absoluta do ar na entrada e saída da amostra; $V_{S}$ é avelocidade do fluido; $L$ é a espessura da amostra; $\mu$ e $\rho$ são a viscosidade a densidade do fluido percolante, respectivamente. $\mathrm{O}$ termo linear da Equação de Forchheimer $\left(\mu \mathrm{V}_{\mathrm{s}} / \mathrm{k}_{1}\right)$ representa as perdas de energia devido ao atrito viscoso e prevalesce em baixas velocidades. $\mathrm{O}$ termo quadrático $\left(\rho \mathrm{V}_{\mathrm{s}}^{2} / \mathrm{k}_{2}\right)$ denota a contribuição da inércia e turbulência na perda de pressão e é mais atuante em altas velocidades.

Os ensaios de secagem foram feitos em um aparato termogravimétrico desenvolvido pelo Grupo de Pesquisa dos Autores, que permite registrar simultaneamente as variações de massa e o perfil de temperatura dentro do forno e na superfície da amostra [9]. Taxas de aquecimento de $10{ }^{\circ} \mathrm{C} / \mathrm{min}$ (taxa de secagem) e $20^{\circ} \mathrm{C} / \mathrm{min}$ (taxa de explosão) foram aplicadas entre $25^{\circ} \mathrm{C} \mathrm{e} 600^{\circ} \mathrm{C}$. As variações de massa foram acompanhadas por meio da derivada parâmetro W em relação ao tempo, que avalia a fração cumulativa de massa perdida durante o aquecimento, em relação ao teor inicial de água na amostra (Equações B e C),

$$
\begin{aligned}
& W(\%)=100 \times \frac{M_{0}-M}{M_{0}-M_{f}} \\
& \left(\frac{d W}{d t}\right)_{i}=\frac{W_{i+1}-W_{i-1}}{t_{i+1}-t_{i-1}}
\end{aligned}
$$

onde: M é a massa instantânea registrada no tempo $\mathrm{t}_{\mathrm{i}}$, durante o aquecimento, $\mathrm{M}_{0}$ é a massa inicial e $\mathrm{M}_{\mathrm{f}}$ a massa final da amostra.

\section{RESULTADOS E DISCUSSÃO}

A Fig. 2 mostra os resultados de medidas de permeabilidade de amostras de concretos refratários elaboradas com diferentes distribuições granulométricas e contendo fibras de polipropileno de diferentes comprimentos. Observa-se inicialmente que as três classes de concretos apresentam níveis de permeabilidade distintos, diferindo entre si aproximadamente por uma ordem de magnitude no valor de $\mathrm{k}_{2}$. Na literatura, essa diferença foi atribuída às proporções entre a quantidade de partículas grossas e finas e o teor de água $[1,4]$. Considere-se formulações elaboradas com as mesmas matérias primas, dentro de uma mesma extensão granulométricas (mesmos $\mathrm{D}_{\max }$ e $\mathrm{D}_{\min }$ ), contendo o mesmo teor volumétrico de água, mas projetadas com diferentes

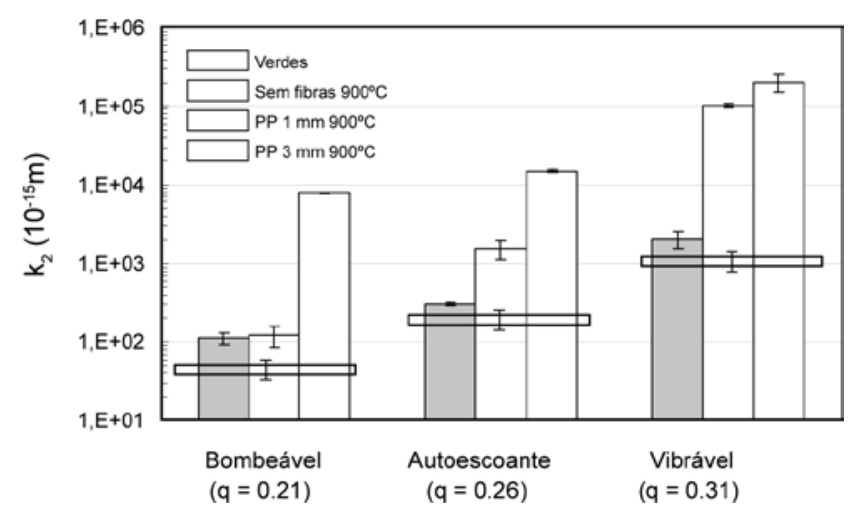

Figura 2: Medidas de permeabilidade $\left(\mathrm{k}_{2}\right)$ de amostras de concretos refratários elaboradas com diferentes distribuições granulométricas e contendo fibras de polipropileno de diferentes comprimentos. [Figure 2: Permeability measurements $\left(k_{2}\right)$ for refractory castables formulation designed with different particle size distribution.] 
coeficientes q do modelo de empacotamento de partículas de Andreasen $(0,17<q<0,37)$. Aquelas composições com maiores valores de $\mathrm{q}(\mathrm{q}>0,26)$ possuem uma maior fração volumétrica de agregados.

Esse fato faz com que nas formulações vibráveis $(q=0,31)$ haja uma maior quantidade de interfaces matriz / agregados e que a concentração volumétrica de sólidos na matriz seja menor, gerando uma fase contínua (matriz) mais permeável nessa classe de concretos. Para as formulações bombeáveis $(\mathrm{q}=0,21)$ e autoescoantes ( $q=0,26)$, ocorre o inverso. Proporcionalmente, há maior quantidade de material fino em relação às formulações vibráveis e maior concentração de sólidos na matriz, fazendo com que a fase contínua, nesses casos, tenha menos interfaces matriz / agregado e partículas melhor empacotadas e mais próximas entre si. Devido a isso, essas classes de concretos apresentam menores valores de permeabilidade e problemas relacionados à secagem mais freqüentes [4].

Observando-se os valores de $\mathrm{k}_{2}$ após o tratamento térmico para as várias formulações contendo fibras de diferentes comprimentos, nota-se que o comprimento mínimo para gerar aumentos de permeabilidade diminui com o aumento do coeficiente q. Para a formulação bombeável $(\mathrm{q}=0,21)$, fibras maiores que $1 \mathrm{~mm}$ são necessárias; para a autoescoante $(\mathrm{q}=0,26)$, o comprimento mínimo está acima de $0,5 \mathrm{~mm}$ e para a vibrável $(\mathrm{q}=0,31)$, ele fica acima de $0,1 \mathrm{~mm}$. Esse comportamento está associado à mínima distância que as interconexões entre as regiões de permeabilidade distinta na estrutura do concreto devem possuir para gerar aumento de permeabilidade $[5,6]$. Para concretos bombeáveis, devido à grande fração volumétrica de matriz, as interfaces permeáveis matriz / agregados ficam mais distantes entre si e necessitam de fibras de maior comprimento para unilas. À medida que a fração volumétrica de finos diminui, as interfaces se aproximam e fibras mais curtas são capazes de efetuar as interconexões.

Nas Figs. 3, 4, 5 e 6 são mostrados os resultados dos testes de secagem dessas formulações. Nos testes de secagem verificou-se que a amostra sem fibras da formulação vibrável secou mais rapidamente que as equivalentes autoescoante e bombeável, sem apresentar explosões quando testada na taxa de aquecimento de $20^{\circ} \mathrm{C} / \mathrm{min}$. Esse fato é um reflexo do elevado nível de permeabilidade inicial que esses materiais apresentam [4]. No entanto, em condições reais, peças maiores (de dezenas a centenas de quilos) produzidas a partir desse tipo de formulação também podem apresentar dificuldades de secagem.

Nas amostras contendo fibras, verificou-se um nível decrescente de benefício gerado pelas fibras com o aumento do coeficiente q: as mudanças nos perfis de secagem causadas pela adição das fibras foram tão mais significativas quanto menor a permeabilidade inicial do concreto. Para a formulação bombeável, a adição de fibras de polipropileno de $3 \mathrm{~mm}$ promoveu um aumento expressivo da taxa de secagem; para a formulação autoescoante e para a vibrável as mesmas fibras pouco alteraram o perfil de secagem.

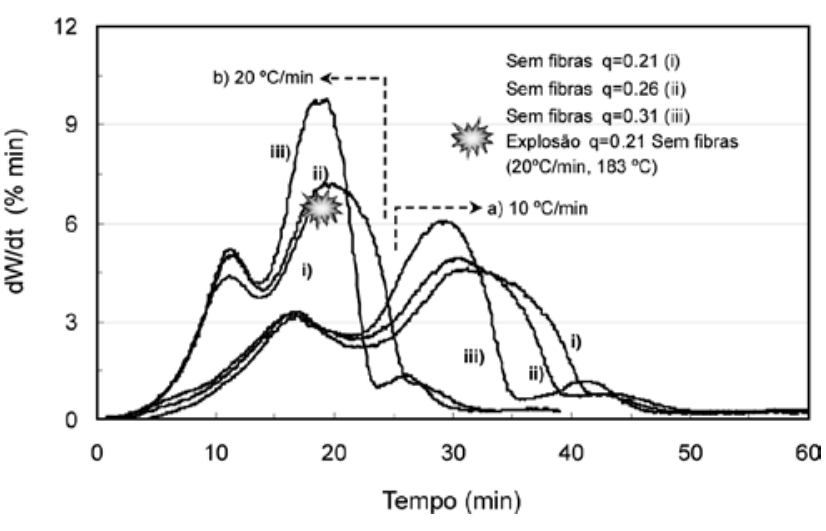

Figura 3: Resultados dos ensaios de a) secagem e b) explosão para amostras de concreto refratário elaboradas com diferentes distribuições granulométricas.

[Figure 3: (a) Drying and (b) explosion tests for refractory castables formulation designed with different particle size distribution.]

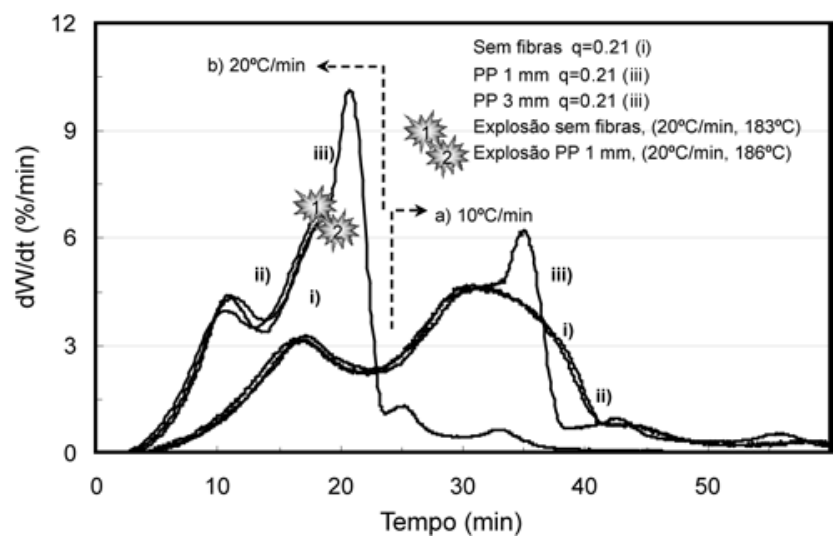

Figura 4: Resultados dos ensaios de a) secagem e b) explosão para amostras de concreto refratário bombeável $(\mathrm{q}=0,21)$, contendo fibras de polipropileno de diferentes comprimentos.

[Figure 4: (a) Drying and (b) explosion tests for pumpable castable formulation $(q=0.21)$, containing different lengths polypropylene fibers.]

Quando se compara o comportamento de secagem das diferentes formulações com fibras com os resultados de medida de permeabilidade, nota-se que, embora a adição de fibras possa aumentar a permeabilidade desses materiais quase que indefinidamente [6], os benefícios na secagem são limitados. A explicação para esse comportamento está associada ao nível de pressurização gerado em cada formulação [7, 9]. Concretos intrinsecamente mais permeáveis (autoescoante e vibrável) desenvolvem menores níveis de pressão interna durante a ebulição. Devido a isso, quando o aumento de permeabilidade ocorre, pouco benefício na taxa de secagem é observado. É importante mencionar que o mecanismo de aumento de permeabilidade, embora pouco solicitado, atuou e mostrou-se capaz de reduzir os potenciais riscos de explosão. 


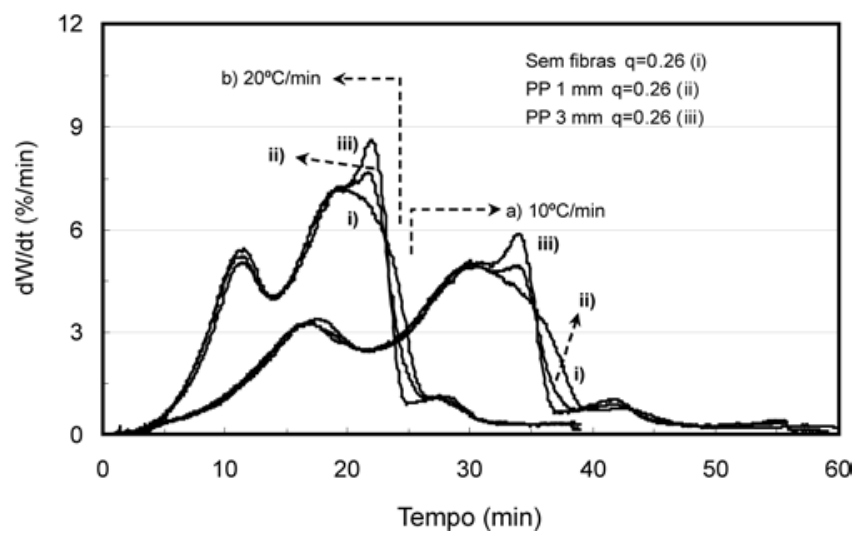

Figura 5: Resultados dos ensaios de a) secagem e b) explosão para amostras de concreto refratário autoescoante $(q=0,26)$, contendo fibras de polipropileno de diferentes comprimentos.

[Figure 5: (a) Drying and (b) explosion tests for self-flowing castable formulation $(q=0.26)$, containing different lengths polypropylene fibers.]

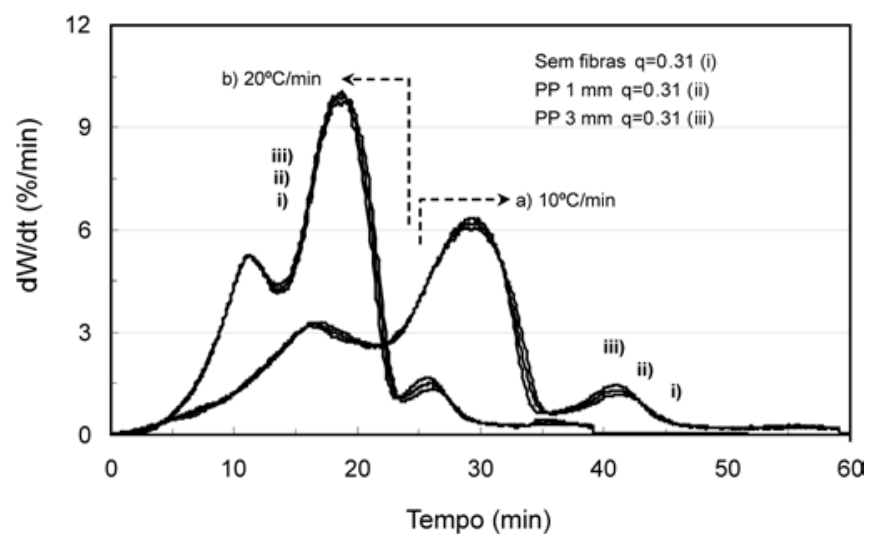

Figura 6: Resultados dos ensaios de a) secagem e b) explosão para amostras de concreto refratário vibrável $(q=0,31)$, contendo fibras de polipropileno de diferentes comprimentos.

[Figure 6: (a) Drying and (b) explosion tests for vibrated castable formulation $(q=0.31)$, containing different lengths polypropylene fibers.]

A existência de níveis críticos de permeabilidade gerados pelas fibras, o mínimo para evitar a explosão e o máximo a partir do qual a secagem pouco se modifica, pode ser atribuída à uma conseqüência da Equação de Forchheimer (Equação A). Essa equação prevê uma relação parabólica entre um gradiente de pressão aplicado em um meio poroso e a vazão resultante de um fluido percolante. Essa relação implica que, em meio muito tortuosos e de baixa porosidade, um determinado gradiente de pressão leva a um certo aumento de vazão. No entanto, se a pressão for continuamente aumentada, acima de um certo nível, a perda de energia resultante do atrito entre o fluido e o meio poroso (relacionado à constante $\mathrm{k}_{1}$ ) e das mudanças de direção e turbulência (relacionadas à constante $\mathrm{k}_{2}$ ), não permite aumentos significativos de vazão e seu valor tende a se estabilizar.
No caso da secagem das composições em estudo, para uma mesma força motriz (pressão de vapor da água em temperaturas acima de $100{ }^{\circ} \mathrm{C}$ ), apresentam-se níveis de permeabilidade distintos. Para a formulação vibrável, a permeabilidade inicial é tão elevada que a adição das fibras pouco contribui para facilitar o escoamento do vapor, embora tenha aumentado significativamente a permeabilidade do material. Para a autoescoante e a bombeável, a permeabilidade inicial é reduzida e caso não seja aumentada, a explosão ocorrerá. No caso das amostras com fibras curtas, o aumento de permeabilidade gerado não é suficiente, levando à pressurização da estrutura e à conseqüente explosão. Aumentando o nível de permeabilidade com maiores teores de fibras e fibras com geometria adequada, o vapor pode ser facilmente removido e a explosão é evitada. Aumentando ainda mais a permeabilidade com teores cada vez maiores de fibras, o mínimo de permeabilidade necessário para evitar a explosão já foi atingido e o elevado valor de $\mathrm{k}_{2}$ pouco contribui para aumentar ainda mais a velocidade do fluido. Esse mecanismo é ilustrado esquematicamente na Fig. 7.

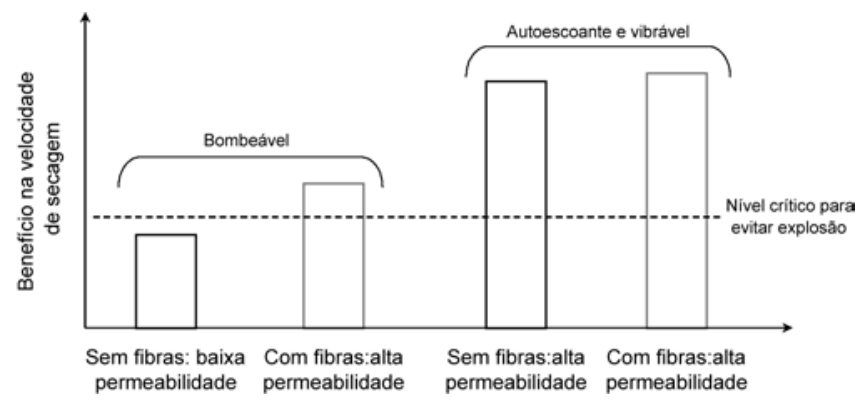

Figura 7: Níveis de permeabilidade proporcionado pelas fibras e a magnitude do benefício equivalente gerado na secagem das diferentes classes de concretos.

[Figure 7: Permeability increase and benefits obtained during drying due to the polypropylene fibers addition.]

$\mathrm{O}$ entendimento das relações entre permeabilidade e o correspondente efeito na taxa de secagem dos concretos adquire grande importância quando outros aspectos do processamento desses materiais são considerados. A adição de fibras muito longas, embora tenha efeito limitado nos benefícios à secagem, pode gerar como efeitos colaterais a redução drástica de fluidez nos concretos e dificuldades de transporte e moldagem [5]. Desse modo, a utilização de fibras curtas (abaixo de $3 \mathrm{~mm}$ ) em concretos bombeáveis e vibráveis se apresenta como uma interessante opção por atuar favoravelmente na secagem, sem afetar significativamente o processamento desses materiais.

\section{CONCLUSÕES}

As fibras de polipropileno atuaram favoravelmente na secagem das diversas classes de concreto refratário 
estudadas. Verificou-se que, dependendo do tipo de concreto empregado, há maior ou menor necessidade da atuação das fibras gerando aumentos de permeabilidade. Para as formulações intrinsecamente mais permeáveis (autoescoante e vibrável), aumentos de permeabilidade de menores magnitudes foram suficientes para reduzir os riscos de explosão; para aquelas menos permeáveis (bombeável), observou-se um nível mínimo de permeabilidade requerido para se evitar a explosão. As relações entre comprimentos iniciais das fibras e os aumentos de permeabilidade gerados indicam que nas composições autoescoante e vibrável a utilização de fibras mais curtas seria mais vantajosa do ponto de vista do processamento desses materiais, pois alia boa atuação como aditivo de secagem com modificações pouco significativas na fluidez desses materiais.

\section{AGRADECIMENTOS:}

Os autores agradecem à FAPESP e ALCOA ALUMÍNIO pelo suporte fornecido a este trabalho e à ALMATIS e ELFUSA pelas matérias primas fornecidas.

\section{REFERÊNCIAS:}

[1] M. D. M. Innocentini, A. R. Studart, R. G. Pileggi, V. C. Pandolfelli, Am. Ceram. Soc. Bull. 80, 5 (2001) 31.

[2] R. G. Pileggi, V. C. Pandolfelli, Am. Ceram. Soc. Bull. 82, 10 (2002).

[3] A. R. Studart, R. G. Pileggi, J. Gallo, V. C. Pandolfelli, Am. Ceram. Soc. Bull. 82, 11 (2002).

[4] M. D. M. Innocentini, R. G. Pileggi, F. T. Ramal Jr., V. C. Pandolfelli, Am. Ceram. Soc. Bull. 83, 7 (2004) 9401.

[5] R. Salomão, F. A. Cardoso, M. D. M. Innocentini, L. R. M. Bittencourt, V. C. Pandolfelli, Am. Ceram. Soc. Bull.

82, 4 (2003) 51-56.

[6] R. Salomão, C. S. Isaac, F. A. Cardoso, M. D. M. Innocentini, V. C. Pandolfelli, Am. Ceram. Soc. Bull. 83, 10 (2003) 931-935.

[7] R. Salomão, V. C. Pandolfelli, J. Tech. Assoc. Refrac. Jpn. 24, 2 (2004) 83-87.

[8] M. D. M. Innocentini, A. R. F. Pardo, V. C. Pandolfelli, J. Am. Ceram. Soc. 85, 6 (2002) 1517-1521.

[9] M. D. M. Innocentini, F. A. Cardoso, M. M. Akyioshy, V. C. Pandolfelli, J. Am. Ceram. Soc. 86, 7 (2003) 1146-1148. (Rec. 30/05/2005, Ac. 21/10/2005) 